\title{
Inklusion zwischen Theorie und Lebenspraxis
}

\author{
Hendrik Trescher \\ Journal für Psychologie, 26(2), 29-49 \\ https://doi.org/10.30820/8248.03 \\ www.journal-fuer-psychologie.de
}

\section{Zusammenfassung}

Ausgehend von einer Unschärfe des Inklusionsdiskurses zielt dieser Beitrag darauf ab, einen Inklusionsbegriff vorzustellen, der Inklusion als Prozess der Dekonstruktion von Diskursteilhabebarrieren versteht. Es wird argumentiert, dass Inklusion und Behinderung zwei wechselseitig aufeinander bezogene Praxen sind, die nur in ihrem jeweiligen Zusammenwirken konsistent gelesen und für Forschung und Praxis handhabbar gemacht werden können. Demnach wäre einem Verständnis von Inklusion, das diese als Prozess der Dekonstruktion von Diskursteilhabebarrieren fasst, ein Verständnis von Behinderung entgegenzustellen, das Behinderung als Praxis bzw. Form des diskursiven Ausschlusses fasst. Im Mittelpunkt des Beitrags steht eine grundlagentheoretische sowie - dem nachgeschaltet - eine empirische Herleitung und Diskussion beider Begriffe, wobei insbesondere deren wechselseitiges Ineinandergreifen hervorgehoben werden soll.

Schlüsselwörter: Inklusion, Behinderung, Diskurs, Subjekt, Subjektivierung, Praxis

\section{Summary}

\section{Inclusion between theory and practice}

Based on the vagueness of the term of inclusion, the present paper aims to introduce a concept of inclusion, which regards inclusion as a process of deconstructing barriers of participation that prevent subjects from participating in discourse. It is argued that inclusion and disability must be considered as two interrelated practices, which can only be consistently understood and finally used in research and practice if viewed in their interrelationship. If inclusion is regarded as a process of deconstructing barriers that prevent subjects from participating in discourse, the concept has therefore to be contrasted with an understanding of disability that refers to disability as a practice respectively as the subject being excluded from discourse. This paper focuses on providing both a theoretical as well as an empirical analysis of both concepts, highlighting their mutual interdependency.

Keywords: inclusion, disability, discourse, subject, subjectivation, practice 


\section{Problemaufriss}

Spätestens seit der Ratifizierung der UN-Behindertenrechtskonvention im Jahr 2009 ist der Begriff Inklusion zur Messlatte sozialpolitischer Reformansätze geworden. Ausdruck findet diese Entwicklung in verschiedenen Projekten und Initiativen, wie sie z. B. in sogenannten Aktionsplänen festgehalten sind. Auf unterschiedliche Art und Weise wird dort die Frage gestellt, wie es - sei es dem Bund, einzelnen Ländern, einer Stadt oder einer Organisation - gelingen kann, Inklusion zu kultivieren und damit einhergehend den (menschenrechtlichen) Anspruch auf gesellschaftliche Teilhabe einzulösen, wie er etwa in der oben aufgeführten UN-Behindertenrechtskonvention formuliert ist. Problematisch ist angesichts dieser Entwicklungen jedoch, dass von theoretischer Seite her keinesfalls geklärt zu sein scheint, was denn unter dem Leitbegriff Inklusion zu fassen und wie das Konzept in die Lebenspraxis zu überführen ist (Ackermann, 2013, S. 171; Cramer \& Harant, 2014, S. 642ff.; Dangl, 2014, S. 258ff.). In diesem Sinne wird der Begriff immer wieder - sei es von sozialpolitischer oder sozialwissenschaftlicher Seite - aufgegriffen und entlang verschiedener theoretischer (z. B. systemtheoretischer oder anthropologisch-normativer) Ansätze (etwa: Budde \& Hummrich, 2013; Degener, 2009; Wansing, 2007; Wocken, 2010) oder mitunter auch - insbesondere in allgemeinöffentlichen Diskursen - ohne theoretische Fundierung ausbuchstabiert. Der Inklusionsbegriff verweilt in einem Raum der Unschärfe (Dannenbeck, 2012; Hinz, 2013), insbesondere dann, wenn es um ein pädagogisch-praktisches Verständnis geht, bzw. dann, wenn ein konkreter Handlungsbezug zur Lebenspraxis hergestellt werden soll. Hierbei fehlt oft der theoretisch-reflexive Bezug. Dies ist problematisch, denn ein theoretisch wie empirisch fundierter Inklusionsdiskurs erscheint letztlich essenziell für eine Disziplin, die sich - in welcher Form auch immer - mit Phänomenen der Ausgrenzung und Marginalisierung beschäftigt. So ist dieser doch maßgeblich für Reflexion und die damit einhergehende begründete Ausrichtung forschungs- sowie auch konkret handlungspraktischer Arbeit. Dem Inklusionsdiskurs wird damit ein zentrales kritischreflexives Moment zuteil, das im Lichte gegenwärtiger Entwicklungen unterzugehen droht (Dannenbeck, 2012, S. 55; Dorrance \& Dannenbeck, 2013, S. 9f.).

Der vorliegende Beitrag knüpft an ebendieser Stelle an. Aufbauend auf den Ergebnissen der Studie Lebensentwürfe von Menschen mit geistiger Behinderung (Trescher, 2017a) soll ein relationaler Inklusionsbegriff vorgestellt werden, der Inklusion als einen Prozess versteht, der auf die Dekonstruktion von Diskursteilhabebarrieren zielt. Inklusion wird dabei als Prozess gedacht, der sich gegenläufig zu Behinderung vollzieht. Behinderung wird wiederum nicht entlang traditioneller Lesarten als vordiskursiver Tatbestand gefasst, sondern es wird vielmehr davon ausgegangen, dass sich Behinderung als Praxis vollzieht - eine Praxis, die Subjekte in ihrer Diskursteilhabe einschränkt und auf diese Weise als behindert hervorbringt (Trescher, 2015, 2017a). Innerhalb die- 
ses Beitrags sollen sowohl der Inklusions- als auch der Behinderungsbegriff - je für sich, jedoch auch in ihrem wechselseitigen Ineinandergreifen - vorgestellt und diskutiert werden. Dabei steht zunächst die Herleitung des Behinderungsbegriffs im Fokus, um hierdurch die Grundlage für die Beschäftigung mit dem Inklusionsbegriff zu schaffen. Diese Herleitung erfolgt zunächst auf grundlagentheoretischer Ebene, indem in Rückbezug auf die Foucault'sche Diskurstheorie (z. B. Foucault, 2003a) gezeigt wird, inwiefern Behinderung als diskursive Praxis verstanden werden kann. In der Folge werden die theoretischen Darlegungen anhand von Auszügen aus der oben genannten empirischen Studie veranschaulicht und vertieft. Ausgehend hiervon wird sich des Inklusionsbegriffes angenommen sowie ein Ausblick gegeben, welche Implikationen sich hieraus für Theorie und Praxis ergeben.

\section{Reformulierung des Behinderungsbegriffs: Behinderung als diskursive Praxis}

Bevor sich eingehender mit der Frage beschäftigt wird, inwiefern Behinderung als diskursive Praxis verstanden werden kann, erscheint es zunächst erforderlich, die hierfür zentralen theoretischen Grundlagen zumindest in Ansätzen zu umreißen. Hierzu zählen einerseits der Foucault'sche Diskursbegriff sowie andererseits die Frage, wie Diskurse dazu beitragen, Subjekte hervorzubringen.

\subsection{Subjektivierung durch Diskurse}

Einsteigend ist festzuhalten, dass Foucault Diskurse als Praktiken auffasst, »die systematisch die Gegenstände bilden, von denen sie sprechen «(Foucault, 1981, S. 74). Diskursen kommt folglich eine performative Wirkmächtigkeit zu, die für das Thema Inklusion von zentraler Bedeutung ist. Diskurse werden von Foucault als »eine Menge von Aussagen « (ebd., S. 156) charakterisiert, die »einem gleichen Formationssystem angehören « (ebd.). Dabei ist hervorzuheben, dass sich Diskurse nicht auf den Faktor Sprache reduzieren lassen: »Zwar bestehen diese Diskurse aus Zeichen; aber sie benutzen diese Zeichen für mehr als nur zur Bezeichnung der Sachen. Dieses mehr macht sie irreduzibel auf das Sprechen und die Sprache « (ebd., S. 74). ${ }^{1}$ Nach Foucault ist es ein zentrales Merkmal von Diskursen, dass sie sich stetig vollziehen, d.h. kein starres Gebilde beschreiben, sondern vielmehr kontinuierlich neu ausgehandelt und hervorgebracht werden (Reckwitz, 2003, S. 298). Einerseits bringen Subjekte Diskurse hervor, indem sie nur in ihnen und durch sie handeln können, andererseits sind es Diskurse, die Subjekte hervorbringen, indem sich das Subjekt eben nur in - ihm durch Diskurse 
zugewiesenen - Subjektpositionen ausdifferenzieren kann. Zuweisung von Subjektpositionen meint dabei vor allem, dass das Subjekt durch den Diskurs in je spezifischer Weise hervorgebracht und anerkannt wird. Praxen der Subjektivierung können damit »(immer auch) als ein Anerkennungsgeschehen « (Ricken, 2013a, S. 84) verstanden werden (Butler, 2014). ${ }^{2}$ In diesem Sinne ist es für ein Subjekt von Bedeutung, ob es als ein Subjekt anerkannt wird, welches im Diskurs als mehr oder weniger handlungsfähig gilt oder nicht. Im Falle von Menschen mit (sog. geistiger) Behinderung kann es also durchaus sein, dass ein Subjekt als Mensch mit (sog. geistiger) Behinderung anerkannt wird und es im Zuge dessen, weil ihm eben gewisse Fähigkeiten nicht qua Status zugesprochen werden, als hilfebedürftiges Subjekt adressiert und hervorgebracht wird, das verstärkter Überwachung und/oder Regulierung durch andere bedarf. Für das Subjekt wird es folglich zur hochgradig relevanten Frage, wie es als was in Diskursen anerkannt wird, wobei ebenfalls deutlich wird, dass die Art und Weise der Anerkennung wiederum gewissen »Normen der Anerkennung « (ebd., S. 34) unterliegt. Der Begriff Subjektivierungspraxen meint im Wesentlichen die Prozesse, »in denen Menschen, Individuen oder Akteure durch den Umgang mit anderem und anderen lernen, sich im Horizont von beziehungsweise in Auseinandersetzung mit spezifischen, naturalen, materialen und sozialen sowie symbolischen Ordnungen als ein Subjekt zu begreifen und zu gestalten « (Ricken, 2013b, S. 34). Aus dieser Perspektive betrachtet sind Subjekt und Diskurs folglich in einem Verhältnis wechselseitiger Hervorbringung zu sehen, welches auch als Verhältnis der »Gleichursprünglichkeit « (Nonhoff \& Gronau, 2012, S. 122) gefasst werden kann. Hieraus folgt, dass sich Subjekt und Diskurs in Relation zum jeweils anderen gleichursprünglich hervorbringen, sodass die Annahme von einem dem Subjekt vorgängigen Diskurs (und umgekehrt) aufzugeben bzw. nicht haltbar ist (Nonhoff \& Gronau, 2012, S. 123; siehe auch Nonhoff, 2014, S. 392).

Bevor nun darauf eingegangen wird, inwiefern sich das Bisherige für eine Reformulierung des Behinderungsbegriffs und die Herleitung eines relationalen Inklusionsbegriffs fruchtbar machen lässt, ist zunächst noch auf einen weiteren Aspekt einzugehen, der hierfür von zentraler Relevanz ist: die Frage nach der Zugänglichkeit von Diskursen bzw. die Frage nach Diskursteilhabe respektive Diskursteilhabebarrieren.

\subsection{Die Zugänglichkeit von Diskursen}

Während der Gesamtdiskurs gewissermaßen die komplette(n) erfahrbare(n) Wirklichkeit(en) umfasst, ist einzelnen Diskursen immer auch ein gewisser Gegenstandsbezug inhärent (Foucault, 2003a, S. 34). Dies wiederum impliziert, dass Diskurse zwangsläufig auch gewisse (gegenstandsbezogene) Grenzen haben, die jedoch (in mehrerlei Hinsicht) von einer Unschärfe geprägt sind (Reckwitz, 2008, S. 11). Es handelt sich bei 
Diskursen insofern um »diskontinuierliche Praktiken [...], die sich überschneiden und manchmal berühren, die einander aber auch ignorieren oder ausschließen« (Foucault, 2003a, S. 34). Folglich gibt es z. B. Themenbereiche und Subjekte, die sich eindeutig einem spezifischen Diskurs zuordnen lassen, und wiederum andere, bei denen dies nicht der Fall ist. Veranschaulicht werden kann dies unter anderem daran, dass in sonderpädagogischen Fachdiskursen z. B. nicht über Quarks oder schwarze Löcher diskutiert wird, sondern über Fragen der Devianz, Stigmatisierung und Marginalisierung. So ist es auch zu erklären, dass ErziehungswissenschaftlerInnen Teil des sonderpädagogischen Fachdiskurses sind und nicht etwa theoretische PhysikerInnen. Parallel hierzu schließen die genannten Fragen des sonderpädagogischen Diskurses unmittelbar an Diskurse anderer Wissenschaftszweige an, z. B. an die der Soziologie oder der Psychologie, sodass es hier zu Überschneidungen kommen kann: Die Grenzen zwischen den einzelnen Diskursen verschwimmen. Anhand des Beispiels zeigt sich auch, dass die Tatsache, dass sich Diskurse voneinander abgrenzen, zwangsläufig auch bedingt, dass Subjekte, die Zugang zu einem je konkreten Diskurs haben, nicht notwendigerweise Zugang zu anderen Diskursen haben. Das Ziehen von Grenzen zwischen den Diskursen erzeugt somit auch immer bestimmte Formen von Ausschluss (ebd., S. 17). Diese Formen des Ausschlusses werden darüber hinaus durch weitere Ausschließungssysteme von Diskursen ergänzt, die ebenfalls dazu dienen, den Zugang zu einem Diskurs bzw. mehreren Diskursen zu regulieren. Foucault spricht hier von inneren und äußeren »Prozeduren der Ausschließung « (ebd., S. 11). Zu den äußeren Prozeduren der Ausschließung gehören z. B. »das verbotene Wort « (ebd., S. 16), womit letztlich eine Festlegung dessen adressiert wird, welche Äußerungen innerhalb eines Diskurses zugelassen werden, sowie die »Ausgrenzung des Wahnsinns « (ebd.), die letztlich auf eine Festlegung dessen rekurriert, wem innerhalb eines Diskurses das Privileg zuteilwird, zu sprechen (siehe ausführlich Trescher, 2015, S. 261ff.). Die inneren Ausschließungssysteme dienen den Diskursen dazu, »ihre eigene Kontrolle selbst ausüben « (Foucault, 2003a, S. 17) zu können, wobei sie »als Klassifikations-, Anordnungs- und Verteilungsprinzipien wirken « (ebd.; siehe ausführlich Trescher, 2015, S. 261ff.). Ihr Ziel ist es, die »Dimension [...] des Ereignisses und des Zufalls « (Foucault, 2003a, S. 17) zu bändigen bzw. eine »Selektion unter den sprechenden Subjekten « (ebd., S. 26) vorzunehmen. Es sind jene äußeren und inneren Ausschließungssysteme, welche die Ordnung des Diskurses (Foucault, 2003a) ausmachen und dazu führen, dass einzelnen Subjekten (entlang der zugewiesenen Subjektpositionen) die Teilhabe an Diskursen gewährt, anderen hingegen die Teilhabe erschwert oder gänzlich verwehrt wird. Die Frage, welche Subjekte in welcher Form an welchen Diskursen ${ }^{3}$ teilhaben (können) und welche Subjekte wiederum durch welche Teilhabebarrieren (manifestiert etwa durch die oben genannten Prozeduren der Ausschließung) in ihrer Teilhabe an je spezifischen Diskursen eingeschränkt werden, ist für das hier zu entfaltende Verständnis von Behinderung und Inklusion von zentraler 
Relevanz. Bedeutsam ist jedoch auch die Unterscheidung zwischen allgemeinen und besonderen Diskursen. Unter allgemeinen Diskursen werden jene Diskurse gefasst, die sich dadurch auszeichnen, dass zunächst keine spezifischen Teilhabevoraussetzungen vorhanden sind, die vonseiten der (potenziellen) DiskursteilnehmerInnen zu erfüllen sind. Allgemeine Diskurse sind insofern jene Diskurse, die prinzipiell jedem Mitglied einer Gesellschaft zugänglich sind respektive innerhalb derer (möglichst) keine Begrenzung der sprechenden Subjekte stattfindet (ebd., S. 26). Hierzu gehört etwa der Zugang zu Informationen, öffentlichen Bereichen oder zu bestimmten Orten im jeweiligen Sozialraum (z. B. Geschäften). Unter besonderen Diskursen werden dementgegen jene Diskurse gefasst, für die spezielle Teilhabevoraussetzungen bestehen - beispielsweise in Form von speziellen Qualifikationen, die den Zugang zum Diskurs reglementieren (Trescher, 2017b). Insofern sind besondere Diskurse solche, in denen eine Verknappung der sprechenden Subjekte explizit vorgesehen ist (siehe ausführlicher: Trescher, 2018). Es zeigt sich, dass es sich bei der Festlegung, welche Diskurse zu allgemeinen und welche zu besonderen Diskursen zu zählen sind, um eine normative, soziokulturell-historisch variable und damit kontingente Praxis handelt. Die Frage nach der Diskursteilhabe von Subjekten - u. a. manifestiert durch die Bestimmung eines Diskurses als allgemein oder besonders - wird damit immer auch zu einer »Frage der Gerechtigkeit, die wiederum immer auch eine politische Frage ist « (Trescher, 2017a, S. 271; siehe auch Forst, 1994, S. 215; 2005, S. 24ff.).

\subsection{Behinderung als diskursive Praxis}

Werden die bisherigen Darstellungen auf die hier relevanten Fragen nach Behinderung und Inklusion übertragen, ergibt sich der folgende Ausgangspunkt: Ein essenzialistischer Blick auf die Kategorie Behinderung, der im Kern davon ausgeht, dass es sich bei Behinderung um eine naturgegebene Form der Abweichung handelt, wird durch eine Perspektive ersetzt, die die Frage nach der Konstitution von Behinderung aufwirft bzw. die Frage danach stellt, wie behinderte Subjekte im Diskurs bzw. in Diskursen hervorgebracht werden. Im Mittelpunkt steht dabei die Frage nach Diskursteilhabebarrieren, die für bestimmte Personen wirkmächtig werden und in der Folge dazu führen, dass sie in ihrer Diskursteilhabe beschränkt und damit als diskursbehindert hervorgebracht werden.

Der hier zu entfaltenden Idee, Behinderung in Anlehnung an Foucault als diskursive Praxis zu fassen, geht eine langjährige Diskussion voraus, die sich um den Behinderungsbegriff sowie die Frage nach dessen Notwendigkeit dreht (Dederich, 2001, S. 122). Ein zentraler wiederkehrender Kritikpunkt besteht im Kern darin, dass der Behinderungsbegriff die Dichotomie behindert/nicht behindert fortschreibt, was insbesondere 
dann zum Problem werden kann, wenn es darum geht, den Versuch zu unternehmen, Behinderung auf theoretischer Ebene auflösbar zu machen (Rösner, 2014, S. 137). Im Laufe der Jahre sind eine Vielzahl von teils expliziten und teils weniger expliziten Vorstellungen davon formuliert worden, was unter Behinderung verstanden werden kann. Grob lassen sich diese Begriffe dabei medizinisch-naturalistischen Perspektiven einerseits und gesellschaftlich-konstruktivistischen Ansätzen andererseits zuordnen (siehe ausführlicher: Trescher, 2017d, S. 13ff.). Das Problem, mit dem viele dieser Behinderungsbegriffe konfrontiert werden, besteht im Wesentlichen darin, dass sie dazu führen, dass der Status »behindert « am Subjekt manifest wird. Aus dieser (mit der Kategorisierung als Mensch mit Behinderung einhergehenden) Statuszuweisung erwachsen wiederum spezifische Praxen des Umgangs, die sich an den als behindert klassifizierten Personen vollziehen (Trescher, 2017c): Dem Subjekt wird durch den Diskurs ein bestimmter Horizont an (behinderten) Subjektpositionen zugewiesen, innerhalb derer es sich herausbilden kann. Vor diesem Hintergrund kann das behinderte Subjekt als » performativer Effekt diskursiver und institutioneller Praktiken « (Rösner, 2014, S. 140) verstanden werden, »die als dichtes Netz von Zuschreibungen die Selbst- und Fremdwahrnehmung einer Gruppe von Individuen hervorbringen « (ebd.). Innerhalb des Diskurses dominiert v. a. ein Blick auf Behinderung, der diese (mal mehr, mal weniger eindeutig) als medizinisches Phänomen und damit im Sinne eines BehindertSeins fasst, was entsprechende Praxen des Umgangs fördert und sich in der Folge - in Anlehnung an die bisherigen Ausführungen - auf die Subjektwerdung der je betroffenen Menschen auswirkt. Veranschaulichen lässt sich dies etwa anhand von DiagnostikManualen wie der International Statistical Classification of Diseases and Related Health Problems (ICD-10). Letztlich erfolgt eine medizinische Erfassung von Behinderung jedoch auch anhand des sogenannten bio-psychosozialen Modells der International Classification of Functioning, Disability and Health (ICF). So werden in Bezug auf Letzteres zwar auch Umweltfaktoren in die Betrachtung miteinbezogen und beispielsweise auch die Problematik der fehlenden persönlichen Handlungsökonomie aus sozialen Gründen reflektiert (Deutsches Institut für Medizinische Dokumentation und Information, 2017). Jedoch wird weiterhin an einem medizinischen Blick (Foucault, 2011) auf Behinderung festgehalten - ist es doch das Ziel der ICF, Krankheit und Behinderung von Menschen technisch zu erfassen, weshalb auch in der ICF letzten Endes immer zwischen behinderten und nicht behinderten Menschen unterschieden wird. Dies hat zwangsläufig zur Folge, dass auch die ICF die Dichotomie fortschreibt und diskursiv reproduziert (Trescher, 2015, S. 18). Von dieser Problematik ist auch das sogenannte soziale Modell von Behinderung betroffen (Oliver, 1990; Waldschmidt, 2006, S. 86), welches Behinderung als »Produkt sozialer Organisation « (Waldschmidt, 2006, S. 86) bzw. als »social creation « (Shakespeare, 2010, S. 268) fasst. Auch hier gibt es einen verbleibenden »essentialistischen Kern« (Waldschmidt, 2006, S. 89), 
der im Endeffekt zur Folge hat, dass Behinderung - nach wie vor - als Problem adressiert wird, »das in irgendeiner Weise der Lösung bedarf « (ebd.). ${ }^{4}$ Diese Problematik kann auch das sogenannte kulturelle Modell von Behinderung - entgegen des selbstgestellten Anspruchs (ebd., S. 91ff.) - nicht überwinden, was v. a. darin begründet liegt, dass es kein Verständnis davon offeriert, wie Behinderung vom Subjekt entkoppelt formuliert werden kann. Anders verhält es sich mit dem Behinderungsbegriff, der hier vorgestellt werden soll und der Behinderung als eine sich vollziehende (immer machtvolle ${ }^{5}$ diskursive Praxis versteht, die sich immer dann vollzieht, » whenever a subject or a group of subjects is partially or fully excluded from (pertinent) discourses by way of powerful practices « (Trescher, 2017b). Dieser Blickwinkel hat zur Folge, dass Behinderung theoretisch auflösbar wäre. Dies wäre dann der Fall, wenn machtvollen Praxen, die als Prozeduren des Ausschlusses bzw. Diskursteilhabebarrieren wirksam werden und spezifische Formen der Subjektivierung bedingen, dekonstruiert würden, wodurch wiederum Formen von Diskursteilhabe und damit auch alternative Formen der Subjektivierung ermöglicht würden. Dem hier zugrunde liegenden Gedanken der Dekonstruktion von Diskursteilhabebarrieren wohnt folglich das Ziel inne, »den Horizont möglicher Identitäten für Menschen mit Behinderungen [zu] erweitern und den Spielraum für die Erprobung alternativer Lebensformen [zu] eröffnen « (Rösner, 2014, S. 141). Eine unmittelbare Konsequenz dieses Verständnisses stellt die Perspektive dar, dass auch Menschen, die bisweilen nicht als behindert bezeichnet wurden, (in bestimmten Situationen) als behindert hervorgebracht werden können - immer dann, wenn sie an Diskursteilhabebarrieren stoßen bzw. durch bestimmte Praxen in ihrer Teilhabe an Diskursen eingeschränkt werden. In diesem Sinne können beispielsweise auch Menschen mit Fluchtmigrationshintergrund als behinderte Subjekte hervorgebracht werden, wenn ihnen in ihrem jeweiligen Zufluchtsland der Zugang zu Sprachkursen und/oder Formen der Beschäftigung verwehrt wird. Gleichzeitig heißt das auch, dass ein Subjekt nur in bestimmten Situationen bzw. Diskursen behindert sein kann, jedoch niemals generell bzw. als Subjekt insgesamt. Insofern wird Behinderung, entlang des hiesigen Verständnisses, als ein je situatives Behindert-Werden verstanden, von dem grundsätzlich jede Person betroffen sein kann und welches von einem (medizinischbiologischen) Behindert-Sein entkoppelt ist. Die Praxis des Behindert-Werdens bezieht sich dabei einerseits auf eine äußere Perspektive, im Sinne der direkten Manifestation von Diskursteilhabebarrieren, wobei die Fragen nach dem (ggf. verwehrten) Zugang zu Diskursen sowie den Diskursen inhärenten Ausschließungssystemen von Bedeutung sind. Relevant ist andererseits aber auch eine nach innen gerichtete Perspektive, d.h. die subjektive Erfahrung des Behindert-Werdens, welche je individuell wahrgenommen und erlebt wird. Insofern ist Behinderung auch das, » was sich als Erfahrung von und mit Behinderung niederschlägt, sich in Biografien und Karrieren verfestigt, was Gegenstand und Bedingung von Anpassungen, Bewältigungsstrategien, Leiden oder 
Widerstand [...] darstellt « (Groenemeyer, 2014, S. 154; siehe auch Shakespeare, 2010, S. 270). Behinderung beschreibt folglich ein komplexes Wechselspiel aus (äußeren) diskursiven Praxen der Ein- und Ausschließung sowie der (inneren) Einschreibung jener Praxen in Subjekte durch Praxen der Subjektivierung. Im weiteren Verlauf soll diese Perspektive anhand von Auszügen einer kürzlich durchgeführten Studie veranschaulicht werden. Hervorzuheben ist dabei, dass es weniger um die komplexe Nachzeichnung des Hervorbringungs- bzw. Entstehungscharakters von Diskursen geht, womit wiederum auch der Begriff des Dispositivs, als » materielle und ideelle Infrastruktur « (Keller, 2005, S. 230) respektive verbindendes bzw. organisierendes Element der Einzelteile der jeweils bezugsrelevanten Diskurse, vertiefend zu berücksichtigen wäre (siehe etwa: Bührmann \& Schneider, 2010, S. 263ff.; 2012, S. 52ff.). Es geht vor allem um die Darstellung und Diskussion von Praxen der (verwehrten) Diskursteilhabe bzw. die damit einhergehenden »Subjektpositionierungen/-formierungen und Subjektivierungsweisen « (Bührmann \& Schneider, 2012, S. 137). Grundsätzlich ist an dieser Stelle jedoch auch noch einmal darauf hinzuweisen, dass Diskursteilhabe mehr als die Problematik des Dispositivs ist, welches bestimmte Personen an bestimmten Positionen im Diskurs reproduziert. Denn Diskursteilhabe meint auch, dass Diskursteilhabe zu bestimmten Diskursen erst ermöglicht werden muss - beispielsweise dann, wenn Menschen, die im Wohnheim leben und dieses kaum verlassen (können), an gewissen Diskursen nicht teilhaben. Es wird also nicht nur von einem Gesamtverständnis Diskurs ausgegangen, sondern auch von kleineren Diskursen (insbesondere solchen, die im Vorangegangenen als allgemeine Diskurse adressiert wurden). Zwar könnte argumentiert werden, dass dieses Außen außerhalb eines Diskurses auch durch ein Dispositiv in einem größeren Diskurs entsteht, dem soll hier jedoch nicht weiter gefolgt werden, da es, wie bereits benannt, vor allem um Beispiele aus der je konkreten Praxis geht. Diskursteilhabebarriere meint also a) zu bestimmten Diskursen nicht zugelassen zu werden (Teilhabebarrieren nach außen) und b) innerhalb von Diskursen (durch - als kontingent gedachte - Dispositive sich in machtvollen Praxen (re-)produzierende Subjektpositionen) nicht die gleichen Teilhabemöglichkeiten zu haben wie andere Subjekte.

\section{Behindert-Werden im Hilfesystem}

Nachdem im Vorangegangenen die theoretische Ausgangsposition dargelegt wurde, wird es im hiesigen Abschnitt darum gehen, diese Ausführungen in Relation zu empirischem Datenmaterial zu setzen und den Behinderungsbegriff (und im darauffolgenden Schritt auch den Inklusionsbegriff) somit auch empirisch herzuleiten. Hierbei soll, unter Bezugnahme auf die Studie Lebensentwürfe von Menschen mit geistiger Behinderung (Trescher, 2017a) ${ }^{6}$, gezeigt werden, wo und inwiefern innerhalb des Systems der Be- 
hindertenhilfe (und darüber hinaus) Praxen angelegt sind, welche die darin betreuten Personen als behindert hervorbringen.

\subsection{Institutionskarrieren}

Eine Behinderungspraxis, die aus fast allen Biografien herausgearbeitet werden konnte, besteht darin, dass sich das Leben vieler Menschen mit sogenannter geistiger Behinderung (noch immer) als (mehr oder weniger stark ausgeprägte) Institutionskarriere fassen lässt (Trescher, 2017a, S. 234). Es konnten Interviews mit Menschen unterschiedlichen Alters geführt werden, die zum Teil mehrere Jahrzehnte in verschiedenen (mal mehr und mal weniger geschlossenen) Einrichtungen der Behindertenhilfe verbracht haben (ebd., S. 135ff.). Komplettiert wird das Leben in exklusiven Einrichtungen in vielen Fällen durch die (vielfach lebenslange) Arbeitstätigkeit in sogenannten Werkstätten für behinderte Menschen (WfbM) oder die (zumindest primäre) Wahrnehmung von behinderungsspezifischen Freizeitangeboten (Trescher, 2015, S. 33ff.). Eine unmittelbare Folge einer solchen biografischen Prägung durch Einrichtungen der Behindertenhilfe dies wurde bei mehreren Personen deutlich - besteht darin, dass es aufseiten der Subjekte zu einer scheinbar konstitutiven Abhängigkeit vom Hilfesystem kommen kann, die zugleich mit einer (teilweise auch tief greifenden) Entfremdung von der allgemeinen Lebenswelt einhergeht. Letzteres ist insbesondere auf die teilweise lebenslängliche räumlich-soziale Distanz zu derselben zurückzuführen, die v. a. (jedoch nicht ausschließlich) stationäre Einrichtungen mit sich bringen. ${ }^{7}$ Für viele der interviewten Personen war ein Leben jenseits des Hilfesystems nicht (mehr) als potenzieller Lebensentwurf vorhanden. Stattdessen beschränkten sich die hervorgebrachten Lebensentwürfe (mehr oder weniger ausschließlich) auf das System der Behindertenhilfe (Trescher, 2017a, S. 236ff.).

\subsection{Erlernte Hilflosigkeit}

Eine weitere Behinderungspraxis, die im Zuge der Studie identifiziert werden konnte, besteht darin, dass das Leben in Betreuungssettings der Behindertenhilfe dazu führen kann, aufseiten der Menschen, die innerhalb der Strukturen leben bzw. durch diese betreut werden, Verhaltensweisen hervorzubringen, die sich auch als erlernte Hilflosigkeit (Seligman, 2004) fassen lassen. Konkret äußert sich diese Behinderungspraxis in einer Adaption der betreffenden Personen an Lebensräume, die nur eingeschränkt persönliche Erfahrungs- und Handlungsspielräume ermöglichen - etwa, weil sie häufig umfassend durch Überwachungs- und Regulierungspraxen gekennzeichnet sind (Trescher, 2017d, S. 160) oder mitunter auch gänzlich von einer Übernahme alltagsprakti- 
scher Aufgaben befreien (Trescher, 2017a, S. 240). Als lebenspraktische Konsequenz jener Adaption konnte unter anderem die Problematik identifiziert werden, dass die betreffenden Personen mitunter die Zuständigkeit für die eigenen Belange mehr oder weniger ganzheitlich an Außenstehende (sprich den Träger einer Einrichtung oder eines Hilfedienstes sowie die dortigen MitarbeiterInnen) abgeben (ebd., S. 240ff.). Es besteht für die jeweiligen Personen häufig schlichtweg nicht (mehr) die Notwendigkeit, sich selbst um die eigenen Belange zu kümmern, woraus letztlich eine gewisse Form von Abhängigkeit sowie lebenspraktischer Unfähigkeit resultiert. Angesichts aktueller Forderungen nach einem Mehr an gesellschaftlicher Teilhabe von Menschen mit sogenannter geistiger Behinderung erscheint diese Behinderungspraxis hochgradig relevant, führt sie doch dazu, von Hilfe- bzw. Unterstützungsleistungen abhängige Subjekte hervorzubringen. Als besonders ausgeprägt und damit letztlich auch besonders wirkmächtig präsentieren sich solche Lebensumstände bei Personen, die in einem erhöhten Maße auf Unterstützungsleistungen angewiesen sind, d. h. bei jenen Menschen, die intensiv betreut werden (ebd., S. 254f.).

\subsection{Bürokratische Überformung}

Eine weitere - quasi allgegenwärtige - Behinderungspraxis ist in der zunehmenden Bürokratisierung des Alltags in Betreuungssettings der Behindertenhilfe zu sehen. Diese Prozesse finden ihren Ausdruck unter anderem in einer Vielzahl von Handlungs- und Ablaufplänen, die strukturierend in den Tag eingreifen bzw. diesen in seinem Ablauf determinieren (wie Dienst-, Dusch-, Tisch-, Einkaufs- und Essenspläne usw.), sowie in zunehmenden Dokumentationspflichten der MitarbeiterInnen (Trescher, 2017d, S. 177f.; 2017e). Es zeigte sich, dass es ebenjene vielfältigen bürokratischen Praxen sind, die im Alltag dazu führen, dass ein pädagogisches Handeln, welches unmittelbar am Subjekt und dessen Interessen anknüpft, immer stärker in den Hintergrund rückt. Dies hat letztlich auch mit mangelnden Zeitkontingenten der Angestellten zu tun: Die akribische Erfassung und Dokumentation von Vorgängen nehmen schlicht einen großen Teil des Arbeitsalltages der MitarbeiterInnen ein, sodass ein pädagogisches (subjektorientiertes) Arbeiten zunehmend erschwert wird (Trescher, 2017d, S. 154). Zentral ist hierbei auch die Problematik, dass ein Handeln, welches sich primär an der Abarbeitung von Plänen ausrichtet bzw. ausrichten muss, nicht die notwendige Flexibilität mitbringt, um ein professionelles pädagogisches ${ }^{8}$ Arbeiten zu gewährleisten. Die Hegemonie bürokratischer Tätigkeiten führt hier zu einer Form von Verbetriebswirtschaftlichung der Angelegenheit Behinderung, die mitunter auch zur Folge hat, dass sich eine (mehr oder wenige umfassende) Entfremdung der (pädagogisch) Tätigen von jenen Personen vollzieht, die ursprünglich im Mittelpunkt des Handelns stehen sollten - im Kontext des 
stationären Wohnens etwa die zu betreuenden Menschen (ebd., S. 185f.). Dies geht wiederum mit tief greifenden Konsequenzen für die betreffenden Subjekte einher. So zeigte sich etwa, dass insbesondere Menschen in stationären Wohnsettings oftmals auf eine enge(re) subjektorientierte Begleitung angewiesen sind - etwa um mit ihnen gemeinsam Interessen herauszuarbeiten, welche wiederum als Ausgangspunkt für eine freizeitliche Betätigung dienen könn(t)en (Trescher, 2015, S. 298ff.). Bei dieser bürokratischen Überformung der Subjekte handelt es sich letztlich um eine Problematik, die nicht ursächlich auf der Ebene einzelner Personen oder Trägerorganisationen angesiedelt ist. Vielmehr handelt es sich um »äußere Vorgaben einer (Behinderung als bürokratisches Problem erfassenden) Gesellschaft und ihrer mit Versorgung reagierenden Subsysteme, welche [...] Behinderung am Subjekt direkt sowie über Vorgaben für die pädagogische Praxis indirekt hervorbringen « (Trescher, 2017d, S. 162). Es zeigt sich hier, dass Behinderungspraxen nicht alleinig auf das System der Behindertenhilfe zurückzuführen sind.

\subsection{Medikalisierung}

Als eine weitere Behinderungspraxis im Leben von Menschen mit sogenannter geistiger Behinderung konnte die Dominanz bzw. Hoheit eines medizinischen Blicks in Betreuungssettings der Behindertenhilfe herausgearbeitet werden (Trescher, 2017a, S. 237ff.). Lebenspraktischen Ausdruck findet diese Behinderungspraxis beispielsweise in der Vergabe von Medikamenten im Falle von sogenannten herausfordernden Verhaltensweisen (Foucault, 2011). Problematisch ist hier mitunter, dass jene herausfordernden Verhaltensweisen vonseiten der MitarbeiterInnen oftmals als Äußerung der als natürlich angenommenen Behinderung gesehen werden. Dies führt dazu, dass alternative Lesarten des Verhaltens - etwa deren Auslegung als eine Form von Auflehnung gegen die bestehenden Strukturen oder auch als Ausdruck anderweitiger persönlicher Krisen (bspw. unverarbeiteter Traumata) - keine Berücksichtigung finden (Trescher, 2017f, 2017g). Die Vergabe von Medikamenten als Antwort auf soziale Probleme, eine Praxis, welche Foucault (2011) als Praxis des medizinischen Blicks kennzeichnet, stellt ein Mittel sozialer Kontrolle dar und ist schlussendlich auch als eine Form der Entmündigung und Regulierung zu kritisieren (Zola, 1972, S. 487; siehe auch Lemke, 2008; Richter \& Hurrelmann, 2016). In diesem Sinne ist es doch gerade in stationären Betreuungssettings das Ziel, durch die Vergabe von verhaltensregulierenden Medikamenten eine Adaption des jeweiligen Subjektes an die gegebenen Strukturen zu erreichen. Das je betreffende Subjekt wird dabei weiterhin in seiner persönlichen Handlungsökonomie beschränkt, entmündigt und mittels eines medikamentösen Zwangs gefügig bzw. im Diskurs sprachlos gemacht. Es lässt sich auch hieran verdeutlichen, dass es nicht 
nur die Strukturen der Behindertenhilfe sind, die für die Hervorbringung behinderter Subjekte verantwortlich sind, sondern schlussendlich auch die Medizin bzw. das Gesundheitssystem, indem es Behinderung bzw. Menschen mit Behinderung zum Gegenstand seiner (Behandlungs-)Praxen macht (Trescher, 2017a, S. 237ff.).

\subsection{Armut}

Die letzte Behinderungspraxis, die hier aufgeführt werden soll, manifestiert sich darin, dass Menschen mit sogenannter geistiger Behinderung in besonderem Maße von Armut betroffen sind (Trescher, 2017a, S. 255). Das Fehlen monetärer Ressourcen war ein Problem, das sich fallübergreifend auf unterschiedlichen Ebenen auf nahezu alle Lebensbereiche auswirkte und damit auch auf unterschiedlichen Ebenen dazu führte, dass Personen als behindert hervorgebracht wurden. Den interviewten Personen war es meist nicht möglich, von dem Lohn, den sie aus ihrer Arbeitstätigkeit (etwa einer $\mathrm{WfbM}$ ) erhalten, auch nur annähernd ein selbstständiges Leben zu führen bzw. auch nur annähernd routinemäßig an allgemeingesellschaftlichen Lebenspraxen teilzuhaben - sei es etwa der halbwegs regelmäßige Besuch des Fitnessstudios, des lokalen Imbisses oder des Kinos. Zur Folge hatte dies ein erhöhtes Maß an Passivität sowie Tendenzen des sozialen Rückzugs. Während dies bereits problematisch erscheint, so wurde ebenfalls deutlich, dass die Behinderungspraxis Armut auch das Fundament für (u.a.) eine weitere Behinderungspraxis legen kann: Vielen der interviewten Personen war es nur deshalb möglich, den oben genannten Aktivitäten nachzugehen oder alltagspraktische Besorgungen, die über den gelegentlichen Erwerb von Süßwaren hinausgehen, zu tätigen, wenn sie zusätzliche finanzielle Unterstützung erhielten, die - im Falle der interviewten Personen - in der Regel durch die Herkunftsfamilie geleistet wurde (ebd., S. $255 \mathrm{ff}$.). Es zeigt sich hier die Komplexität der Behinderungspraxis Armut, denn letztlich führt die Rückbindung an die Herkunftsfamilie, wie sie hier als punktueller Ausweg aus der Armut gewählt wird, dazu, dass Ablösungsprozesse und damit auch Selbstermächtigungspraxen, welche sich ohnehin oftmals problematisch gestalten (Trescher, 2015, S. 212), weiterführend behindert werden. Armut trägt insofern auf unterschiedlichen Ebenen mit dazu bei, dass die betreffenden Personen als abhängige, behinderte Subjekte hervorgebracht werden.

\section{Zum Inklusionsbegriff: Inklusion als dekonstruktive Praxis}

Ziel der vorangegangenen Ausführungen war es, anhand einiger kurzer Beispiele zu zeigen, wie Subjekte, die als geistig behindert klassifiziert werden, innerhalb des Diskurses 
bestimmten (geistig behinderten) Subjektpositionen zugewiesen werden und wie sie mit den damit einhergehenden Lebenskontexten in ihrer Diskursteilhabe eingeschränkt und in der Folge auf unterschiedliche Art und Weise als behindert hervorgebracht werden (können). Als eine zentrale Erkenntnis kann angesehen werden, dass gerade Menschen mit sogenannter geistiger Behinderung sich in ihrem Leben vielfältigen Behinderungspraxen ausgesetzt sehen, wenngleich hervorzuheben ist, dass die Wirkmächtigkeit von Behinderungspraxen nicht zu generalisieren, sondern vielmehr im Lichte der je konkreten Lebenspraxis zu reflektieren ist (Trescher, 2017g, S. 49ff.). Insofern lässt sich sagen, dass sich bereits aus den hier dargelegten Auszügen die Notwendigkeit ergibt, Behinderung als komplexes Zusammenspiel unterschiedlicher Praktiken zu betrachten. Einem relationalen Verständnis von Behinderung ist insofern auch ein relationales Verständnis von Inklusion gegenüberzustellen. Wird Behinderung, wie hier vorgeschlagen, als diskursive Praxis verstanden, die sich immer dann vollzieht, wenn Subjekte an Diskursteilhabebarrieren stoßen (z. B. durch Faktoren wie erlernter Hilflosigkeit, bürokratischer Überformung oder Armut), wäre Inklusion zwangsläufig als jene Praxis zu fassen, die auf die Dekonstruktion ebenjener Diskursteilhabebarrieren zielt (Trescher, 2017a, S. 183ff.). Mit Dekonstruktion ist hier eine Praxis des Infragestellens gemeint, welche »Ambivalenzen und Widersprüche aufdeckt « (Zima, 2016, S. 1; siehe auch Saar, 2007, S. 167), »eine Bejahung der Differenz und des Differierens « (Zirfas, 2001, S. 50) beinhaltet und im Wesentlichen darauf abzielt, »das Denken von innen her für das zu öffnen, was dieses seit jeher ausgeschlossen hat, um dem anderen gerecht werden zu können « (ebd.). Die übergeordnete Zielsetzung ist dabei, »das Vorherrschende [...] in seiner gesellschaftlichen Funktion zur Aufrechterhaltung der herrschenden Norm(alität) sowie in seinen Konstitutions- und Konstruktionsprozessen zu untersuchen, dabei Ausgeschlossenes zu erkennen und Hierarchisierungen aufzuweichen « (Hartmann, 2001, S. 80f.). Das hier vorgeschlagene Verständnis von Inklusion ist demnach nicht als eine moralische Idee zu verstehen, die postuliert, dass alle Menschen an allen (allgemeinen und besonderen) Diskursen teilhaben sollen. Stattdessen geht es darum, Inklusion als eine der Behinderung gegenläufige Praxis zu verstehen, die sich - ebenso wie Behinderung - immer relational und hochgradig komplex gestaltet. In Anbetracht dessen muss sich auch von dem Gedanken distanziert werden, dass sich Inklusion mittels einer einzelnen Maßnahme oder auf Grundlage einer einfachen Ursache-Wirkungs-Logik herbeiführen lässt. Es geht letztlich darum, Inklusion als komplexe - nicht nur auf Menschen mit sog. Behinderung fokussierte - Praxis zu erfassen, die im Kern auf Diskursverschiebungen abzielt, welche sich wiederum zwangsläufig in der Lebenspraxis vollziehen müssen (Trescher, 2017a, S. 183). Hierdurch ist Inklusion auch immer hochgradig krisenhaft für alle Beteiligten, geht es doch gerade auch darum, dass bestehende Konventionen und Routinen, wie z. B. gewisse Sittlichkeitsnormen, aufgebrochen und durch neue ersetzt werden müssen (Trescher, 2018). 
Letztlich ist Inklusion jedoch auch für die bisher ausgeschlossenen Subjekte krisenhaft, da Diskursverschiebung beispielsweise auch die Überwindung potenzieller (Berührungs-) Ängste und Unsicherheiten bedeutet. Von zentralem Stellenwert sind hier v.a. Diskursverschiebungen, die auf eine Dekonstruktion der zu Beginn aufgezeigten Prozeduren des Ausschlusses und damit auf eine Öffnung von Sprecherrollen abzielen. $\gg$ Niemand kann in die Ordnung des Diskurses eintreten, wenn er nicht gewissen Erfordernissen genügt, wenn er nicht von vorneherein dazu qualifiziert ist « (Foucault, 2003a, S. 26). Ausschlussmechanismen, die bestimmte Subjekte pauschal von der Teilhabe an (insbesondere allgemeinen) Diskursen ausschließen und sie damit in gewisser Weise sprachlos machen, sind daher im Zeichen von Inklusion durch Mechanismen zu ersetzen, die auf eine Vergrößerung des Kreises der sprechenden Subjekte abzielen. Im hiesigen Kontext beinhaltet dies insbesondere Praxen der Ermächtigung - Ermächtigung dahingehend, dass Subjekte befähigt werden, sich Diskurse anzueignen bzw. in Diskursen zu sprechen. Bezugnehmend auf die vorangegangenen empirischen Auszüge können hier etwa die Behinderungspraxen der bürokratischen Überformung, der Institutionskarrieren und der erlernten Hilflosigkeit als Beispiele angeführt werden, die Subjekte pauschal von einer Teilhabe an (insbesondere allgemeinen) Diskursen ausschließen und einer auf Diskursteilhabe ausgerichteten Qualifizierung diametral gegenüberstehen. Unmittelbar hieran angeknüpft ist folglich auch die Dekonstruktion gouvernementaler Regierungspraxen, die Subjekte in ihrer persönlichen Handlungsökonomie begrenzen (Trescher, 2017a, S. 48f.).

Relevant ist jedoch nicht nur der Zugang zu Diskursen und die Fähigkeit, dort zu sprechen, sondern ebenso die Frage, wer innerhalb der entsprechenden Diskurse gehört wird und wer nicht. Relevant erscheinen hier - im Sinne von Inklusion - Diskursverschiebungen, die beispielsweise an der »Ausgrenzung des Wahnsinns « (Foucault, 2003a, S. 16) ansetzen. Im Sinne Foucaults gilt jenes Subjekt als wahnsinnig, »dessen Diskurs nicht ebenso zirkulieren kann wie der der andern: sein Wort gilt für null und nichtig, es hat weder Wahrheit noch Bedeutung « (ebd., S. 12). Dass Äußerungen von Menschen mit sogenannter geistiger Behinderung (sogar) in Betreuungssettings der Behindertenhilfe oftmals nur wenig Gehör finden können, konnte anhand der Ausführungen zur Praxis der Medikalisierung veranschaulicht werden, mittels derer (häufig nonverbale) Äußerungen im Rahmen einer Fremdauslegung übergangen und mittels der Verabreichung sogenannter ruhigstellender Medikationen eingeebnet werden. In diesen Fällen führt der Status Mensch mit sogenannten herausfordernden Verhaltensweisen verstärkt dazu, dass die Äußerungen des je betreffenden Subjekts als irrelevant und als Ausdruck einer als natürlich konstruierten geistigen Behinderung gesehen werden (Trescher, 2017a, S. 237ff.). Es gilt insofern, in Bezug auf diese konkreten Praxen, Räume dafür zu schaffen, dem Wort von Menschen mit sogenannter geistiger Behinderung - sowohl in Diskursen der Behindertenhilfe, jedoch auch auf allgemein- 
gesellschaftlicher Ebene - (mehr) Gewicht zu verleihen. Hierzu gehört letztlich auch eine Dekonstruktion dessen, was Foucault als »Wille zur Wahrheit « (Foucault, 2003a, S. 15) bezeichnet, d. h. die Frage, was innerhalb eines Diskurses thematisiert wird und was nicht (ebd.). Inklusion bezieht sich in diesem Zusammenhang folglich auch auf die Dekonstruktion normalisierender Diskurspraktiken, welche mittels der Formulierung von Normen »eine Scheidung in normal und anormal erlauben und in sozialen und institutionellen Praktiken operieren « (Lemke, 2014, S. 96f.; siehe auch Foucault, 1976, S. 123).

\section{Abschließende Bemerkungen}

Wird der Blick abschließend auf eine stärker handlungspraktische Ebene gerichtet, lässt sich feststellen, dass es für eine Dekonstruktion der hier vorgestellten Behinderungspraxen notwendig erscheint, insbesondere im Kontext von Betreuungssettings der Behindertenhilfe, verstärkt auf eine umfassende Refokussierung des Subjekts hinzuwirken (Trescher, 2017d, S. 179ff.). Es muss in erster Linie darum gehen, das Subjekt in seiner je spezifischen Lebenssituation zu erfassen und pädagogische Interventionen entsprechend auszurichten. Hierfür erscheint es jedoch zuvorderst geboten, pädagogische Praxis als solche überhaupt erst zu ermöglichen, indem z. B. Praxen der bürokratischen Überformung dekonstruiert werden (Trescher, 2017e). Insgesamt ist es essenziell, einen kritischen Blick zu entwickeln, der auch die Frage berücksichtigt, wann Unterstützungsmaßnahmen als Behinderungspraxis wirkmächtig werden und wie hierauf zu reagieren ist. Insbesondere hierfür ist es im Kontext pädagogischer Betreuungssettings notwendig, eine kritisch-reflexive Praxis zu etablieren, welche einen verstehenden $\mathrm{Zu}$ gang wählt. Hierbei sollte es weniger um die Frage nach gestellten Diagnosen oder Ähnlichem gehen, sondern v. a. darum, das je konkrete Subjekt sowie den Kontext bzw. die Gruppe in den Fokus zu rücken (Trescher, 2017a, S. 264f.). Eine zentrale Aufgabe kommt an diesem Punkt auch der Wissenschaft zu, ist es doch - anschließend an die im Beitrag vorgestellten Überlegungen - v. a. ihre Aufgabe, mittels kritischer Forschung Behinderungspraxen offenzulegen und diese damit einer Reflexion zugänglich zu machen. Hiervon ausgehend ist es möglich, theoriegeleitet Praxen der Dekonstruktion zu formulieren, welche wiederum in die Praxis selbst zurückgespiegelt und mit dieser diskutiert werden (können) (ebd., S. 269f.). Die Aufgabe von Wissenschaft und Forschung ist insofern auf das Bereitstellen von Ergebnissen und Perspektiven beschränkt, da es der Praxis letztlich offensteht,

»welchen Gebrauch sie von den Forschungsergebnissen und Ergebnissen der Erkenntniskritik in ihren je konkreten Entscheidungen macht. Die Wissenschaft kann nicht mehr 
tun, als in möglichst großer Klarheit und argumentativer, methodischer Stringenz die wissenschaftlich erweisbaren Konsequenzen einer Entscheidung zu explizieren. Aber die Entscheidung selbst zu treffen, ist sie in keiner Weise kompetent «(Oevermann, 1996, S. 104).

Erneut hervorzuheben ist abschließend, dass Inklusion selbstredend nicht als im klassischen Sinne behinderungsspezifisch zu verstehen, sondern ebenso wie Behinderung (als Praxis) von gesamtgesellschaftlicher Relevanz und damit auch als gesamtgesellschaftliche Aufgabe zu betrachten ist. Insofern befassen sich die hier präsentierten Überlegungen, welche sich auf die Lebenssituation von Menschen mit sogenannter geistiger Behinderung beschränken, lediglich mit einer Facette unter vielen. Eine zentrale Frage von Inklusion ist, welche Personen, in welcher Form, Zugang zu welchen Diskursen haben bzw. welche Teilhabebarrieren bzw. Prozeduren des Ausschlusses ihre Teilhabe an entsprechenden Diskursen verhindern und sie damit als in spezifischer Art und Weise behindert hervorbringen. Diese Frage gilt es auch auf andere Bereiche des gesellschaftlichen Lebens zu übertragen. Zentral sind im Zuge dessen gerade auch die Fragen, ob und in welchem Rahmen es den Personen gestattet ist, innerhalb der jeweiligen Diskurse zu sprechen, und welche Subjekte in welcher Form innerhalb der Diskurse gehört werden. Denn gerade in dem Moment des Sprechens im Diskurs liegt ein Moment der Widerständigkeit bzw. die Möglichkeit zur Veränderung des Diskurses, werden Diskurse doch - wie dargestellt - erst durch Subjekte hervorgebracht, die sich Diskurse aneignen (Butler, 2013; Zima, 2017, S. 363).

\section{Anmerkungen}

1 Hervorzuheben ist an dieser Stelle, dass der Idee der nicht-diskursiven Praktiken nicht gefolgt wird, denn »[d]ie sprachlichen und nicht-sprachlichen Elemente werden nicht bloß nebeneinander gestellt, sondern konstituieren ein differentielles und strukturiertes System von Positionen, das heißt einen Diskurs« (Laclau \& Mouffe, 2006, S. 145; siehe auch Bührmann \& Schneider, 2008, S. 43ff.; Foucault, 2003a, S. 396).

2 Aufgenommen wird an dieser Stelle eine Lesart von Anerkennung, die sich v. a. an die Auseinandersetzungen von Ricken (2006; 2009; 2013a) bzw. Balzer und Ricken (2010) anlehnt. Eine solche Lesart richtet sich gegen die vielfach geläufige Auslegung, nach der Anerkennung einseitig als »ein wertschätzendes und unterstützendes Handeln des Akzeptierens und Respektierens (Ricken, 2009, S. 88) zu fassen wäre, welches als »verfügbares Mittel« (Ricken, 2006, S. 222) von den Akteurlnnen innerhalb einer Interaktion entweder verweigert oder erteilt werden könne. Anerkennung wird hier eher als konstitutives Moment von Interkation begriffen, d. h., statt die Frage zu stellen, ob ein Individuum anerkannt wird oder nicht, erscheint hier die Frage relevanter, als was ein Subjekt anerkannt und auf welche Art und Weise es damit im Diskurs hervorgebracht wird. Dies hat zur Folge, dass Anerkennung - entlang des hiesigen Verständnisses - v.a. zu einem subjekttheoretisch-analytischen Begriff wird, im Kontext der Frage nach der sozio-kulturell- 
historischen Konstitution von Subjektivität, wodurch die moralphilosophische Dimension von Anerkennung in den Hintergrund tritt.

3 Deutlich wird hier, dass sich Diskursteilhabe und Diskursteilhabebarrieren nicht auf den (Gesamt-)Diskurs beziehen, sondern vielmehr auf je spezifische Diskurse.

4 Mitunter wird dies auch durch die Vertreterlnnen des sozialen Modells selbst kritisiert, wie bspw. Shakespeare (2010), wenn er ausführt: »The social model's benefits as a slogan and political ideology are its drawbacks as an academic account of disability« (S. 269). Shakespeare geht dabei sogar auch so weit festzustellen, dass »the social model has now become a barrier to further progress« (Shakespeare, 2010, S. 272), weshalb er seine Weiterentwicklung fordert (Shakespeare, 2010, S. 272f.).

5 Macht ist gemäß diesem Verständnis »nicht etwas, was man erwirbt, wegnimmt, teilt, was man bewahrt oder verliert« (Foucault, 2003b, S. 94) und ist somit nichts, das (ggf. einzelnen) Personen oder Institutionen innewohnt. Macht ist, nach Foucault, vielmehr produktiv und vollzieht sich in der Praxis als Netz von diskursiven Beziehungen - »weil sie von überall kommt, ist die Macht überall« (Foucault, 2003b, S. 94).

6 Die als Lehrforschungsprojekt angelegte Studie wurde im Sommersemester 2015 und Wintersemester 2015/16 an der Goethe-Universität Frankfurt durchgeführt. Grundlage des Projekts war die Erhebung und, entlang der Verfahren der objektiven Hermeneutik (Oevermann, 2000), rekonstruktive Auswertung von 16 biografischen Interviews im Kontext sog. geistiger Behinderung. Die Interviewpartnerlnnen wurden entlang des Paradigmas der größtmöglichen Kontrastivität ausgewählt. Als relevante Faktoren wurden hier bspw. Alter, Geschlecht, Wohn- und Beschäftigungssituation gewertet. Der zentrale Fokus des Projekts bestand einerseits in der Auseinandersetzung mit der vergangenen und gegenwärtigen Lebenssituation, andererseits jedoch auch mit den (weiteren) Lebensentwürfen und Zukunftsperspektiven von Menschen, die als geistig behindert gelten (siehe vertiefend: Trescher, 2017a). Von besonderem Interesse war z. B. die Frage danach, wie sich der Alltag der betreffenden Personen gestaltet bzw. in der Vergangenheit gestaltet hat. Auch die Rolle der familiären Herkunft, anderweitiger Sozialkontakte sowie der je spezifischen Wohn- und Arbeitsbedingungen wurden in den Blick genommen.

7 In diesem Zusammenhang konnte bspw. festgestellt werden, dass auch das ambulant betreute Wohnen hier nicht zwangsläufig eine Ausnahme darstellt (Trescher, 2017a, S. 124).

8 Rekurriert wird an dieser Stelle auf einen Professionsbegriff nach Oevermann (1996, 2002), der pädagogisches Handeln in erster Linie als interaktives, reflexives Handeln versteht, welches wiederum eine »Beziehungspraxis« (Oevermann, 1996, S. 115) mit den Betreuten ermöglicht.

\section{Literatur}

Ackermann, K. (2013). Geistigbehindertenpädagogik zwischen Disziplin und Profession. In K. Ackermann, O. Musenberg \& J. Riegert (Hrsg.), Geistigbehindertenpädagogik!? Disziplin - Profession - Inklusion (S. 171-184). Oberhausen: Athena-Verlag.

Balzer, N. \& Ricken, N. (2010). Anerkennung als pädagogisches Problem - Markierungen im erziehungswissenschaftlichen Diskurs. In A. Schäfer \& C. Thompson (Hrsg.), Anerkennung (S. 35-87). Paderborn: Ferdinand Schöningh.

Budde, J. \& Hummrich, M. (2013). Reflexive Inklusion. Zeitschrift für Inklusion, 7(4). http://www.inklusion -online.net/index.php/inklusion-online/article/view/193/199 (29.03.2018).

Bührmann, A. \& Schneider, W. (2010). Die Dispositivanalyse als Forschungsperspektive. Begrifflichkonzeptionelle Überlegungen zur Analyse gouvernementaler Taktiken und Technologien. In 
J. Angermüller \& S. van Dyk (Hrsg.), Diskursanalyse meets Gouvernementalitätsforschung. Perspektiven auf das Verhältnis von Subjekt, Sprache, Macht und Wissen (S. 261-288). Frankfurt am Main: Campus.

Bührmann, A. \& Schneider, W. (2012). Vom Diskurs zum Dispositiv. Eine Einführung in die Dispositivanalyse (2. Aufl.). Bielefeld: transcript.

Butler, J. (2013). Psyche der Macht. Das Subjekt der Unterwerfung (7. Aufl.). Frankfurt am Main: Suhrkamp. Butler, J. (2014). Kritik der ethischen Gewalt. Erweiterte Ausgabe. Frankfurt am Main: Suhrkamp.

Cramer, C. \& Harant, M. (2014). Inklusion - Interdisziplinäre Kritik und Perspektiven von Begriff und Gegenstand. Zeitschrift für Erziehungswissenschaft, 17(4), 639-659.

Dangl, O. (2014). Inklusion als Herausforderung für die Geistigbehindertenpädagogik. Zeitschrift für Bildungsforschung, 4(3), 257-270.

Dannenbeck, C. (2012). Wie kritisch ist der pädagogische Inklusionsdiskurs? Entpolitisierungsrisiko und theoretische Verkürzung. In K. Rathgeb (Hrsg.), Disability Studies. Kritische Perspektiven für die Arbeit am Sozialen (S. 55-67). Wiesbaden: VS.

Dederich, M. (2001). Menschen mit Behinderung zwischen Ausschluss und Anerkennung. Bad Heilbrunn: Klinkhardt.

Degener, T. (2009). Menschenrechte und Behinderung. In M. Dederich \&W. Jantzen (Hrsg.), Behinderung und Anerkennung (S. 160-169). Stuttgart: Kohlhammer.

Deutsches Institut für Medizinische Dokumentation und Information (DMDI) (2005). Internationale Klassifikation der Funktionsfähigkeit, Behinderung und Gesundheit. http://www.dimdi.de/static/de/ klassi/icf/index.htm (29.03.2018).

Dorrance, C. \& Dannenbeck, C. (2013). Doing Inclusion. Zur Einführung in den Band. In C. Dorrance (Hrsg.), Doing Inclusion. Inklusion in einer nicht inklusiven Gesellschaft (S. 9-12). Bad Heilbrunn: Klinkhardt.

Forst, R. (1994). Kontexte der Gerechtigkeit. Politische Philosophie jenseits von Liberalismus und Kommunitarismus. Frankfurt am Main: Suhrkamp.

Forst, R. (2005). Die erste Frage der Gerechtigkeit. APuZ. Aus Politik und Zeitgeschichte, (37), 24-31.

Foucault, M. (1976). Die Macht und die Norm. In M. Foucault (Hrsg.), Mikrophysik der Macht. Über Strafjustiz, Psychiatrie und Medizin (S. 114-123). Berlin: Merve.

Foucault, M. (1981). Archäologie des Wissens. Frankfurt am Main: Suhrkamp.

Foucault, M. (2003a). Die Ordnung des Diskurses (9. Aufl. Mit einem Essay von Ralf Konersmann). Frankfurt am Main: Fischer.

Foucault, M. (2003b). Der Wille zum Wissen. Frankfurt am Main: Suhrkamp.

Foucault, M. (2011). Die Geburt der Klinik. Eine Archäologie des ärztlichen Blicks (9. Aufl.). Frankfurt am Main: Fischer.

Groenemeyer, A. (2014). Soziale Praxis - Institutionen - Diskurse - Erfahrung: Behinderung im Problematisierungsprozess. Soziale Probleme, 25(2), 150-172.

Hartmann, J. (2001). Bewegungsräume zwischen Kritischer Theorie und Poststrukturalismus. Eine Pädagogik vielfältiger Lebensweisen als Herausforderung für die Erziehungswissenschaft. In A. Tervooren, J. Hartmann, B. Fritzsche \& A. Schmidt (Hrsg.), Dekonstruktive Pädagogik. Erziehungswissenschaftliche Debatten unter poststrukturalistischen Perspektiven (S. 65-84). Opladen: Leske + Budrich.

Hinz, A. (2013). Inklusion - von der Unkenntnis zur Unkenntlichkeit?! - Kritische Anmerkungen zu einem Jahrzehnt Diskurs über schulische Inklusion in Deutschland. Zeitschrift für Inklusion, 7(1). http://www.inklusion-online.net/index.php/inklusion-online/article/view/26/26 (29.03.2018).

Keller, R. (2005). Wissenssoziologische Diskursanalyse. Grundlegung eines Forschungsprogramms. Wiesbaden: VS.

Laclau, E. \& Mouffe, C. (2006). Hegemonie und radikale Demokratie. Zur Dekonstruktion des Marxismus. Wien: Passagen Verlag. 
Lemke, T. (2008). Gouvernementalität und Biopolitik. Wiesbaden: VS.

Lemke, T. (2014). Eine Kritik der politischen Vernunft. Foucaults Analyse der modernen Gouvernementalität (6. Aufl.). Hamburg: Argument.

Nonhoff, M. \& Gronau, J. (2012). Die Freiheit des Subjekts im Diskurs. Anmerkungen zu einem Verhältnis der Gleichursprünglichkeit. In R. Keller, W. Schneider \& W. Viehöver (Hrsg.), Diskurs - Macht - Subjekt. Theorie und Empirie von Subjektivierung in der Diskursforschung (S. 109-130). Wiesbaden: VS.

Nonhoff, M. (2014). Subjekt. In D. Wrana, A. Ziem, M. Reisigl, M. Nonhoff \& J. Angermuller (Hrsg.), DiskursNetz. Wörterbuch der interdisziplinären Diskursforschung (S. 391-393). Frankfurt am Main: Suhrkamp.

Oevermann, U. (1996). Theoretische Skizze einer revidierten Theorie professionalisierten Handelns. In A. Combe \& W. Helsper (Hrsg.), Pädagogische Professionalität. Untersuchungen zum Typus pädagogischen Handelns (S. 70-182). Frankfurt am Main: Suhrkamp.

Oevermann, U. (2000). Die Methode der Fallrekonstruktion in der Grundlagenforschung sowie der klinischen und pädagogischen Praxis. In K. Kraimer (Hrsg.), Die Fallrekonstruktion. Sinnverstehen in der sozialwissenschaftlichen Forschung (S. 58-156). Frankfurt am Main: Suhrkamp.

Oevermann, U. (2002). Professionalisierungsbedürftigkeit und Professionalisiertheit pädagogischen Handelns. In M. Kraul, W. Marotzki \& C. Schweppe (Hrsg.), Biographie und Profession (S. 19-63). Bad Heilbrunn: Klinkhardt.

Oliver, M. (1990). The politics of disablement. London: Macmillan.

Reckwitz, A. (2003). Grundelemente einer Theorie sozialer Praktiken. Eine sozialtheoretische Perspektive. Zeitschrift für Soziologie, 32(4), 282-301.

Reckwitz, A. (2008). Unscharfe Grenzen. Perspektiven der Kultursoziologie. Bielefeld: transcript.

Richter, M. \& Hurrelmann, K. (2016). Die soziologische Perspektive auf Gesundheit und Krankheit. In M. Richter \& K. Hurrelmann (Hrsg.), Soziologie von Gesundheit und Krankheit (S. 3-22). Wiesbaden: VS.

Ricken, N. (2006). Erziehung und Anerkennung. Vierteljahresschrift für wissenschaftliche Pädagogik, 82(2), 215-230.

Ricken, N. (2013a). Anerkennung als Adressierung. Über die Bedeutung von Anerkennung für Subjektivationsprozesse. In T. Alkemeyer, G. Budde \& D. Freist (Hrsg.), Selbst-Bildungen. Soziale und kulturelle Praktiken der Subjektivierung (S. 69-100). Bielefeld: transcript.

Ricken, N. (2013b). Zur Logik der Subjektivierung. Überlegungen an den Rändern eines Konzepts. In A. Gelhard, T. Alkemeyer \& N. Ricken (Hrsg.), Techniken der Subjektivierung (S. 29-47). München: Wilhelm Fink.

Ricken, N. (2009). Über Anerkennung. Spuren einer anderen Subjektivität. In N. Ricken, H. Röhr, J. Ruhloff, K. Schaller (Hrsg.), Umlernen (S. 75-92). München: Wilhelm Fink.

Rösner, H. (2014). Behindert sein - behindert werden. Texte zu einer dekonstruktiven Ethik der Anerkennung behinderter Menschen. Bielefeld: transcript.

Saar, M. (2007). Beschreiben/Zersetzen: Dekonstruktion als Institutionskritik. In A. Niederberger \& M. Wolf (Hrsg.), Politische Philosophie und Dekonstruktion. Beiträge zur politischen Theorie im Anschluss an Jacques Derrida (S. 165-180). Bielefeld: transcript.

Seligman, M. E.P. (2004). Erlernte Hilflosigkeit (3. Aufl.). Weinheim: Beltz.

Shakespeare, T. (2010). The Social Model of Disability. In L.J. Davis (Hrsg.), The disability studies reader (3. Aufl., S. 266-273). London: Routledge.

Trescher, H. (2015). Inklusion. Zur Dekonstruktion von Diskursteilhabebarrieren im Kontext von Freizeit und Behinderung. Wiesbaden: Springer VS.

Trescher, H. (2017a). Behinderung als Praxis. Biographische Zugänge zu Lebensentwürfen von Menschen mit sgeistiger Behinderungr. Bielefeld: transcript.

Trescher, H. (2017b). Disabling practices. Cogent Social Science, 3(1). https://www.tandfonline.com/doi/ full/10.1080/23311886.2017.1328771?scroll=top\&needAccess=true (29.03.2018). 
Trescher, H. (2017c). Von behindernden Praxen zu einer Reformulierung des Behinderungsbegriffs. Behindertenpädagogik, 56(3), 267-282.

Trescher, H. (2017d). Wohnräume als pädagogische Herausforderung. Lebenslagen institutionalisiert lebender Menschen mit Behinderung (2. Aufl.). Wiesbaden: Springer VS.

Trescher, H. (2017e). Zur bürokratischen Überformung der Subjekte. Wohnen in der stationären Alten- und Behindertenhilfe. In M. Meuth (Hrsg.), Pädagogisch institutionelles Wohnen (S. 245-266). Wiesbaden: Springer VS.

Trescher, H. (2017f). Behinderung, Fluchtmigration, Kommunikation. Teilhabe, 56(4), 150-155.

Trescher, H. (2017g). Lebensentwürfe von Menschen mit »geistiger Behinderung». Behinderte Menschen, $40(1), 47-52$.

Trescher, H. (2018/im Druck). Inklusion als Kritik. Dekonstruktivistische Zugänge am Beispiel der ıVersorgung von Menschen mit geistiger Behinderung in Deutschland. Zeitschrift für Inklusion.

Waldschmidt, A. (2006). Brauchen die Disability Studies ein »kulturelles Modell« von Behinderung? In G. Hermes \& E. Rohrmann (Hrsg.), Nichts über uns - ohne uns! (S. 83-96). Neu-Ulm: AG SPAK.

Wansing, G. (2007). Behinderung. Inklusions- oder Exklusionsfolge? Zur Konstruktion paradoxer Lebensläufe in der modernen Gesellschaft. In A. Waldschmidt \& W. Schneider (Hrsg.), Disability Studies, Kultursoziologie und Soziologie der Behinderung. Erkundungen in einem neuen Forschungsfeld (S. 275-298). Bielefeld: transcript.

Wocken, H. (2010). Integration \& Inklusion. Ein Versuch, die Integration vor der Abwertung und die Inklusion vor Träumereien zu bewahren. In A. Stein, S. Krach \& I. Niediek (Hrsg.), Integration und Inklusion auf dem Weg ins Gemeinwesen. Möglichkeitsräume und Perspektiven (S. 204-234). Bad Heilbrunn: Klinkhardt.

Zima, P.V. (2016). Die Dekonstruktion (2. Aufl.). Tübingen: Francke.

Zima, P.V. (2017). Theorie des Subjekts. Subjektivität und Identität zwischen Moderne und Postmoderne. Tübingen: Francke.

Zirfas, J. (2001). Identitäten und Dekonstruktionen. Pädagogische Überlegungen im Anschluss an Jacques Derrida. In A. Tervooren, J. Hartmann, B. Fritzsche \& A. Schmidt (Hrsg.), Dekonstruktive Pädagogik. Erziehungswissenschaftliche Debatten unter poststrukturalistischen Perspektiven (S. 49-63). Opladen: Leske + Budrich.

Zola, I. K. (1972). Medicine as an Institution of Social Control. Sociological Review, 20(4), 487-504.

\section{Der Autor}

Hendrik Trescher, Prof. Dr. phil. habil., hat seit 01.10.2018 die Professur für Erziehungswissenschaften mit dem Schwerpunkt Inklusion und Exklusion an der PhilippsUniversität Marburg inne. Seine Forschungsschwerpunkte sind: Inklusionsforschung, Partizipation von Menschen mit Behinderungen, Pädagogik bei kognitiven Beeinträchtigungen (sog. »geistige Behinderung « und »Demenz «), Methoden qualitativer Sozialforschung, sozialwissenschaftliche (insbesondere institutionssoziologische) Grundlagen der (Sonder-)Pädagogik, Disability Studies, Subjekt- und Diskursforschung. Er wurde unter anderem 2016 ausgezeichnet mit dem Forschungspreis der Deutschen Interdisziplinären Gesellschaft zur Förderung der Forschung für Menschen mit geistiger Behinderung für das Buch: Inklusion. Wiesbaden, Springer VS, 2015.

Kontakt: hendrik.trescher@uni-marburg.de 